\title{
Preparation of electrochemically reduced graphene oxide/multi-wall carbon nanotubes hybrid film modified electrode, and its application to amperometric sensing of rutin
}

\author{
SULING YANG ${ }^{\mathrm{a}}$, GANG LI ${ }^{\mathrm{a}}$, MEIFANG HU ${ }^{\mathrm{a}}$ and LINGBO QU ${ }^{\mathrm{a}, \mathrm{b}, *}$ \\ ${ }^{a}$ College of Chemistry and Chemical Engineering, Anyang Normal University, Anyang 455002 , \\ People's Republic of China \\ ${ }^{\mathrm{b}}$ Chemistry and Chemical Engineering School, Henan University of Technology, Zhengzhou 450001 , \\ People's Republic of China \\ e-mail: yang_suling@163.com; qulingbo@zzu.edu.cn
}

MS received 8 February 2014; revised 15 April 2014; accepted 22 April 2014

\begin{abstract}
Through a facile electrochemical method, we prepared an electrochemically reduced graphene oxide (ERGO)/multi-wall carbon nanotubes (MWNTs) hybrid film modified glassy carbon electrode (GCE), and characterized it by Fourier transform infrared spectroscopy (FTIR), scanning electron microscopy (SEM) and x-ray diffraction (XRD) The experimental results demonstrated that ERGO-MWNTs/GCE exhibited excellent electrocatalytic activity toward rutin as evidenced by the significant enhancement of redox peak currents in comparison with a bare GCE, ERGO/GCE and MWNTs/GCE. This method has been applied for the direct determination of rutin in real samples with satisfactory results.
\end{abstract}

Keywords. Multi-wall carbon nanotubes; grapheme; rutin; electroanalyisis

\section{Introduction}

Rutin is one of the most abundant bioactive flavonoid, also called rutoside. As a natural flavone derivative, rutin has many physiological activities, such as antioxidant, hemostat, anti-inflammatory, anti-tumor, and anti-bacterial. ${ }^{1-3}$ It is often used as a clinical drug to strengthen capillaries and helps in treating easy bruising or bleeding of the skin. ${ }^{4}$ Scientists have been increasingly paying more attention to the development of the sensitive, economical, convenient and efficient detection of rutin. Many techniques have been developed for the detection of rutin, such as capillary electrophoresis, ${ }^{5}$ chemiluminescence, ${ }^{6}$ flow injection analysis, ${ }^{7}$ high-performance liquid chromatography (HPLC) ${ }^{8}$ and spectrophotometry. ${ }^{9}$ Nevertheless, some of the above mentioned methods are time-consuming, costly, and need complicated pre-processing, as well as professional technicians. Electrochemical detection is characterized by low-cost instrumentation, small dimensions, high sensitivity, simplicity and on-site monitoring. Different modified electrodes have been developed for the determination of rutin because it is an electroactive compound. ${ }^{10,11}$ However, higher selective

\footnotetext{
*For correspondence
}

and more sensitive electrochemical techniques for the detection of rutin should be developed.

Graphene (GR), as a novel two-dimensional material, has attracted tremendous attention due to its remarkable characteristics, such as high specific surface area, electrical conductivity, chemical stability and good biocompatibility. The application of graphene in the field of electrochemical sensing had been reviewed. ${ }^{12}$ Graphene is hydrophobic. Especially, it tends to form irreversible agglomerates or even restocks to form graphite owing to strong $\pi-\pi$ interaction and van der Waals interactions, ${ }^{13}$ which is a limit for its further application. In general, graphene can be largescale synthesized by chemical reduction of graphene oxide (GO) using different kinds of reducing agents, such as sodium borohydride and hydrazine hydrate. ${ }^{14,15}$ Recently, by cyclic voltammetry $(\mathrm{CV})^{16}$ and constant potential method, ${ }^{17}$ a simple, fast and green electrochemical reduction of GO was developed to prepare graphene, which is suitable for the graphene modified electrode. ${ }^{18,19}$

On the nanoscale, carbon nanotubes (CNTs) have attracted tremendous interest since their discovery in $1991 .^{20}$ Recent studies implied that CNTs used as electrode material can provide strong electrocatalytic activity. For example, CNTs can accelerate electron transfer of various molecules on the surface of electrode 
and increase the available electroactive surface area of various electroactive substances. ${ }^{21}$ Graphene is provided with excess of $15,000 \mathrm{~cm}^{2} \mathrm{~V}^{-1} \mathrm{~s}^{-1}$ electron mobility at room temperature but has problem of its agglomerates property between individual graphene sheets, and results in lower conductivity. To overcome the above-mentioned disadvantages, intermingling of graphene nanosheets with other carbon based nanostructures has been proposed. ${ }^{22}$ The introduction of CNTs not only effectively inhibits the aggregation and restacking of graphene nanosheets, but also supplies a higher surface area and more electroactive interaction sites. $^{23}$

In this paper, we prepared ERGO-MWNTs/GCE by $\mathrm{CV}$. Experiments revealed that the redox peak currents of rutin could be remarkably enhanced on ERGO-MWNTs/GCE. In addition, The ERGOMWNTs hybrids modified electrode could effectively separate the overlapping voltammetric response of rutin and ascorbic acid (AA) into two well-defined voltammetric peaks, which suggested that the ERGOMWNTs/GCE could be used to determine rutin in the presence of a certain concentration of AA.

\section{Experimental}

\subsection{Reagents and solutions}

AA and rutin were purchased from National Institute for the Control of Pharmaceutical and Biological Products, China. Phosphate buffer solution (PBS) was prepared by mixing $0.1 \mathrm{M} \mathrm{NaH}_{2} \mathrm{PO}_{4}$ and $0.1 \mathrm{M} \mathrm{Na}_{2} \mathrm{HPO}_{4}$ at different rations to adjust the $\mathrm{pH}$ value of 4.0. The Rutin Tablets and Compound Rutin Tablets were commercially available, and the labeled amount of rutin is $20 \mathrm{mg} /$ tablet. Spectral graphite (about $30 \mu \mathrm{m}$, Shanghai Colloid Chemical Plant, China) used to synthesize GO, was obtained from Shanghai Carbon Co., Ltd. The multi-wall carbon nanotubes (diameter: 10-20 nm, length: 1-2 $\mu \mathrm{m}$, purity $>95 \%$ ) were obtained from Shenzhen Nanotech Port Co., Ltd. China. Nafion (wt. $5 \%$ ) was purchased from Sigma. All the other chemicals used in this study were all of analytical grade. All experiments were completed in a supporting electrolyte of PBS (0.1 M, pH 4.0) at room temperature $\left(25 \pm 1^{\circ} \mathrm{C}\right)$.

\subsection{Apparatus}

CHI 660E electrochemical workstation (Shanghai Chenhua Instrument Co., Ltd. Shanghai, China) was performed for all the electrochemical measurements. A conventional three-electrode system was used, including a bare GCE $(d=3 \mathrm{~mm})$ or ERGO-MWNTs/GCE as working electrode, an $\mathrm{Ag} / \mathrm{AgCl}(3 \mathrm{M})$ as reference electrode and a platinum wire electrode as auxiliary electrode. The morphology of the modified films was characterized using SEM (SEM, Hitachi S-4800 field emission scanning electron microscope (http://www. hitachi-hta.com). All the $\mathrm{pH}$ values were measured with a PHS-3C precision $\mathrm{pH}$ meter (Leici Devices Factory of Shanghai, China), which was calibrated with standard buffer solution every day.

\subsection{Preparation of ERGO-MWNTs/GCE}

Firstly, GO was synthesized from spectral graphite by a modified Hummers' method. ${ }^{24}$ Secondly, the pretreated GCE and the acidified MWNTs were obtained according to our previous report, ${ }^{25}$ and then, in $10.0 \mathrm{~mL} 0.1 \%$ $(w / w)$ Nafion methanol solution, adequate amount of GO and MWNTs were sonicated together for $30 \mathrm{~min}$. to get $0.5 \mathrm{mg} \mathrm{mL}^{-1} \mathrm{GO}-\mathrm{MWNT}$ homogeneous solution. Subsequently, 5.0 $\mu \mathrm{L}$ GO-MWNTs suspension was coated on the pretreated GCE evenly and dried at room temperature to get GO-MWNTs/GCE. Finally, in $0.1 \mathrm{M} \mathrm{NaH}_{2} \mathrm{PO}_{4}$ solution, the GO-MWNTs/GCE was reduced at a potential range of $-1.6-0 \mathrm{~V}$ at $80 \mathrm{mV} \mathrm{s}^{-1}$ for $10 \mathrm{~min}$. After reduction, the electrode surface was rinsed with ethanol and double-distilled water in turn, and dried with $\mathrm{N}_{2}$ to achieve ERGO-MWNTs/GCE. GO/GCE, GRGO/GCE and MWNTs/GCE were prepared in a similar procedure.

\subsection{Experimental procedure for electrochemical analysis}

Before each measurement, the three-electrode system was installed in a blank solution, and cycled successively for three times at the potential of $-0.2-0.8 \mathrm{~V}$ (vs. $\mathrm{Ag} / \mathrm{AgCl}$ ). The quantitative determination of rutin was achieved by measuring the oxidation peak current after background subtraction using differential pulse voltammetry (DPV). Five tablets of Compound Rutin Tablets or Rutin Tablets was ground into powder in an agate mortar, respectively. And then the average weight of one tablet was accurately weighed and dissolved in $10 \mathrm{~mL}$ ethanol. After ultrasonic dissolving for an hour, the mixtures were filtrated. In order to fit into the linear range of the proposed method, appropriate amount of the filtrate was diluted by a factor of $1 / 10,000(\mathrm{v} / \mathrm{v})$ with 0.1 M PBS ( $\mathrm{pH} 4.0$ ) for the measurement. The dilution process can actually help decreasing the matrix effect in real samples. 


\section{Results and discussion}

\subsection{Characterization of modified electrode}

As shown in figure 1, the morphologies of different electrodes were characterized by SEM. The bare GCE presented a uniform and smooth surface (figure 1a). An obvious homogeneous membrane appeared on the ERGO/GCE (figure 1b), and the thin layer of ERGO sheets displayed a typical crumpled and folded structure. The morphology of the MWNTs/GCE (figure 1c) showed a network-like structure. Compared to the MWNTs/GCE, nanocomposite film of ERGOMWNTs (figure 1d) exhibited the long tortuous network of MWNTs covered with the thin ERGO sheets completely, implying that ERGO and MWNTs evenly mixed together. The results may be the $\pi-\pi$ stacking interaction between the hydrophobic regions of ERGO and the side walls of MWNTs, which avoided the aggregation of ERGO and kept the ERGO-MWNTs dispersing stably for long time.

The FTIR spectra for the pristine MWNTs, GO, GO-MWNTs and ERGO-MWNTs were shown in figure 2. $\mathrm{C}=\mathrm{O}$ band characteristic of ketone/quinone on MWNTs was observed at $\sim 1635 \mathrm{~cm}^{-1}$, and the band of $\sim 1197 \mathrm{~cm}^{-1}$ was most probably due to the $\mathrm{C}-\mathrm{O}$ stretching deformation vibration (figure $2 \mathrm{a}$ ). ${ }^{26}$ The spectrum of GO displayed three broad and intense peaks centered at around $\sim 1050 \mathrm{~cm}^{-1}, \sim 1623 \mathrm{~cm}^{-1}$ and $\sim 1735 \mathrm{~cm}^{-1}$

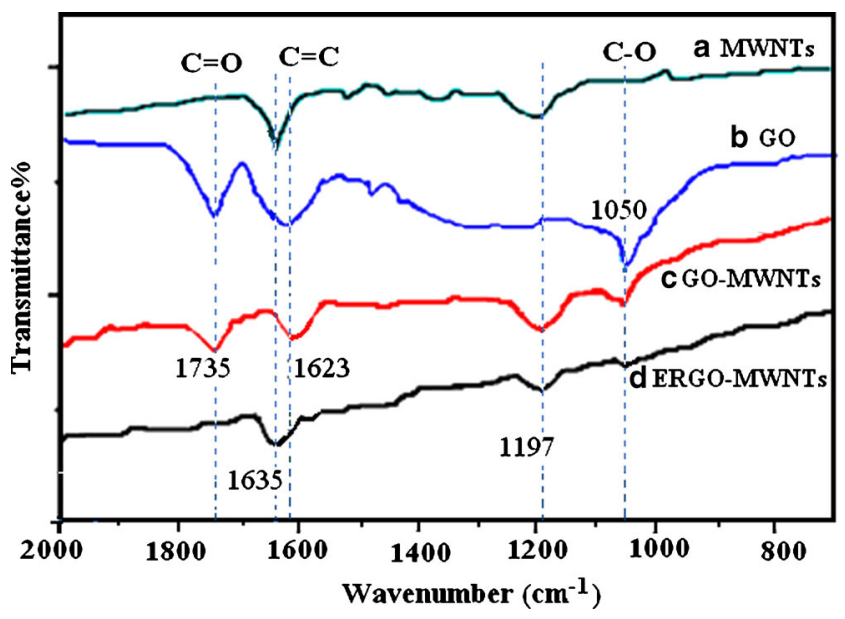

Figure 2. FTIR spectra of (a) MWNTs, (b) GO, (c) GOMWNTs and (d) ERGO-MWNTs.

(figure $2 \mathrm{~b}$ ), while the band at $\sim 1050 \mathrm{~cm}^{-1}$ could be due to the $\mathrm{C}-\mathrm{O}$ stretching mode of epoxy or alkoxy, and $\mathrm{C}=\mathrm{O}$ in carboxylic acid and carbonyl moieties (v(carbonyl)) at $1735 \mathrm{~cm}^{-1} ; \mathrm{C}=\mathrm{C}$ at $\sim 1623 \mathrm{~cm}^{-1}$ assigned to skeletal vibrations of unoxidized graphitic domains or contribution from the stretching deformation vibration of intercalated water. ${ }^{17}$ Interestingly, the spectra of GO-MWNTs hybrid material exhibited all the characteristic peaks of GO and MWNTs despite the lower peak intensity (figure 2c). Apparently, these peaks appeared due to the co-existence of GO along
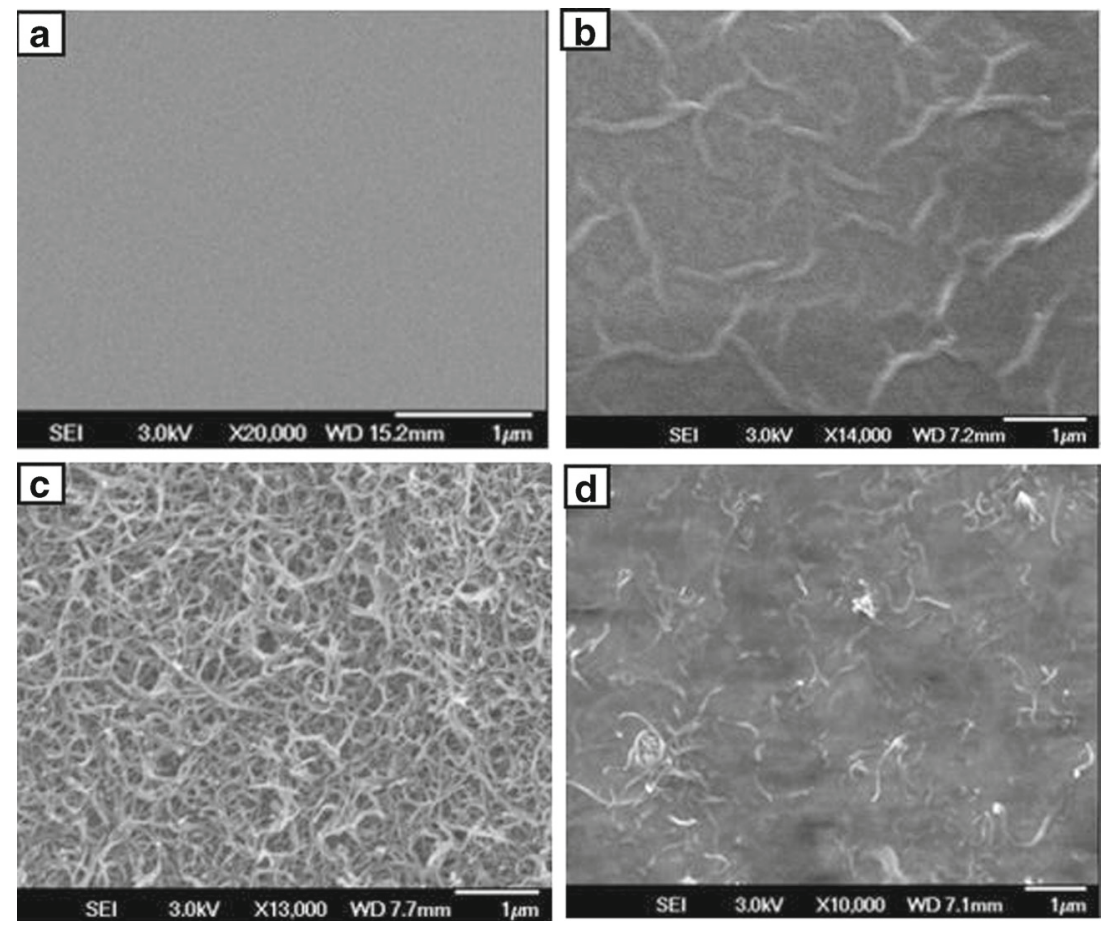

Figure 1. SEM images of different electrodes: (a) bare GCE, (b) ERGO/ GCE, (c) MWNTs/GCE and (d) ERGO-MWNTs/GCE. 
with MWNTs in the hybrid. As the GO was electrochemically reduced (figure $2 \mathrm{~d}$ ), the adsorption bands of $\mathrm{C}=\mathrm{O}$ in carboxylic acid and carbonyl moieties at $\sim 1735 \mathrm{~cm}^{-1}$ disappeared; the peak of $\mathrm{C}-\mathrm{O}$ stretching mode of epoxy or alkoxy at $\sim 1050 \mathrm{~cm}^{-1}$ and the peak at $\sim 1623 \mathrm{~cm}^{-1}$ were considerably decreased, which confirmed the successful conversion of GO into ERGO. ${ }^{17}$

XRD could further support the characterization of GO and ERGO. XRD patterns of the GO and ERGO were recorded in figure 3 . The feature diffraction peak of GO appeared at $10.1^{\circ}(002)$ as the $\mathrm{AB}$ stacking order was observed in graphite oxide with layer-to-layer distance ( $d$-spacing) of $0.849 \mathrm{~nm}$. After the GO was electrochemically reduced, the diffraction peak of ERGO was at $26.4^{\circ}$, similar to the reported results. ${ }^{27}$

\subsection{Electrochemical reduction of GO-MWNTs}

Figure 4 shows the electrochemical reduction of GOMWNTs film at the potential range from $0 \mathrm{~V}$ to $1.6 \mathrm{~V}$ in $0.1 \mathrm{M} \mathrm{NaH}_{2} \mathrm{PO}_{4}$. It can be seen that during the first cycle, a large cathodic peak appeared at $-1.15 \mathrm{~V}$ due to the reduction of oxygen functional groups such as hydroxyl, epoxy and carboxyl groups residing on the surface of $\mathrm{GO},{ }^{28}$ which proved the electrochemical reduction of GO to form ERGO-MWNTs.

\subsection{Voltammetric behaviour of rutin at different electrodes}

Figure 5 demonstrated CVs responses of the $8.0 \times$ $10^{-5} \mathrm{M}$ rutin at different electrodes in PBS ( $\mathrm{pH} 4.0$ ). It was found that the oxidation peak current $\left(i_{\mathrm{pa}}\right)$ of rutin at the ERGO/GCE (c) and MWNTs/GCE (d) were obviously higher than that of at the GO/GCE (b),

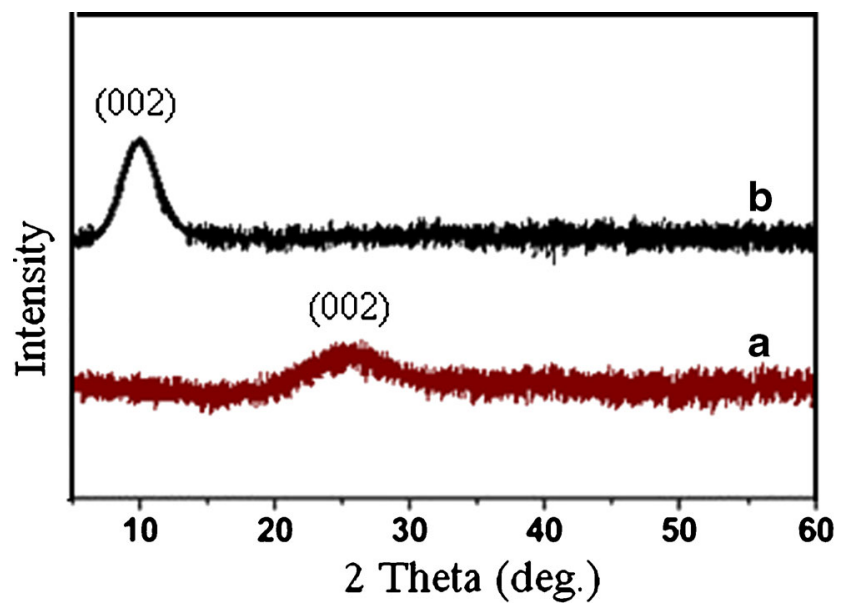

Figure 3. X-ray diffraction patterns of the (a) ERGO (b) and pristine $\mathrm{GO}$.

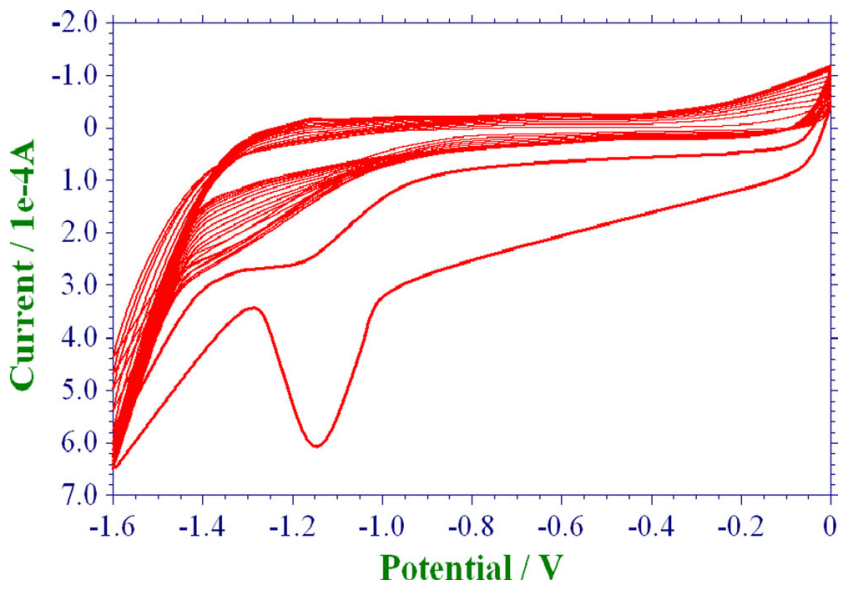

Figure 4. Electrochemical reduction of GO-MWNTs hybrid for 14 cycles in $0.1 \mathrm{M} \mathrm{NaH}_{2} \mathrm{PO}_{4}$ ( $\mathrm{pH} 4.7$ ); scan rate $80 \mathrm{mV} \mathrm{s}^{-1}$.

GO-MWNTs/GCE (e) and GCE (f). Compared to the ERGO/GCE and MWNTs/GCE, the redox peak currents at the ERGO-MWNTs/GCE (a) were remarkably higher. The experimental results might be attributed to the synergistic effect of the 3D nano-structured ERGOMWNTs, including its large specific surface area and remarkable electric conductivity. The optimal ERGOMWNTs/GCE showed the highest capacitative current value over the other control systems. It is related to the successful conversion of GO into ERGO. The excellent $\mathrm{sp}^{2}$ hybridized structure of ERGO contributes to the high capacitive nature, which promotes the electron gain and loss of ions during charging/discharging. ${ }^{29}$ In addition, this capacitance behaviour can be explained by that ERGO provides a high electrical conductivity and high specific surface area, allowing rapid and effective electron and ion transport. ${ }^{30,31}$

\subsection{Effect of scan rate on the response of rutin at ERGO-MWNTs/GCE}

Figure 6 a showed the CVs of $8.0 \times 10^{-5} \mathrm{M}$ rutin at the ERGO-MWNTs/GCE. In the range of $20-300 \mathrm{mV}$ $\mathrm{s}^{-1}$, the redox peak current enhanced gradually along with the increase of the scan rate. The linear relationship of the anodic peak current and the scan rate $(v)$ is corresponding to a regression equation: $i_{\mathrm{pa}}=0.0391 v+1.7356\left(i_{\mathrm{pa}}: \mu \mathrm{A}, v: \mathrm{mV} \mathrm{s}^{-1}\right.$, $r=0.9978$ ) (figure 6b), which implied that the system presented features corresponding to an adsorptioncontrolled process of rutin at the ERGO-MWNTs/GCE. According to the reported paper, ${ }^{32}$ the adsorption of rutin on ERGO-MWNTs/GCE includes chemisorbed and physisorbed rutin is moieties. Concerned about the chemisorption, it may be that rutin is immobilized 


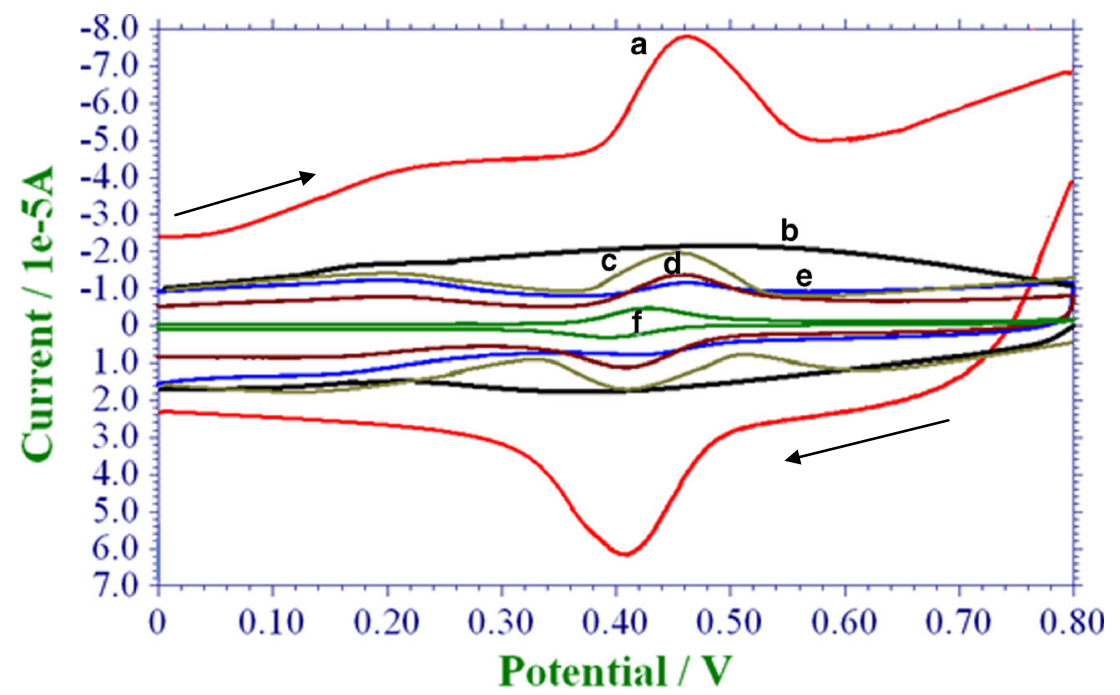

Figure 5. CVs of (a) ERGO-MWNTs/GCE, (b) GO/GCE, (c) ERGO/GCE, (d) MWNTs/GCE, (e) GO-MWNTs/GCE and (f) GCE in 0.1 M PBS (pH 4.0) containing $8.0 \times 10^{-5} \mathrm{M}$ rutin with a scan rate of $80 \mathrm{mV} \mathrm{s}^{-1}$.
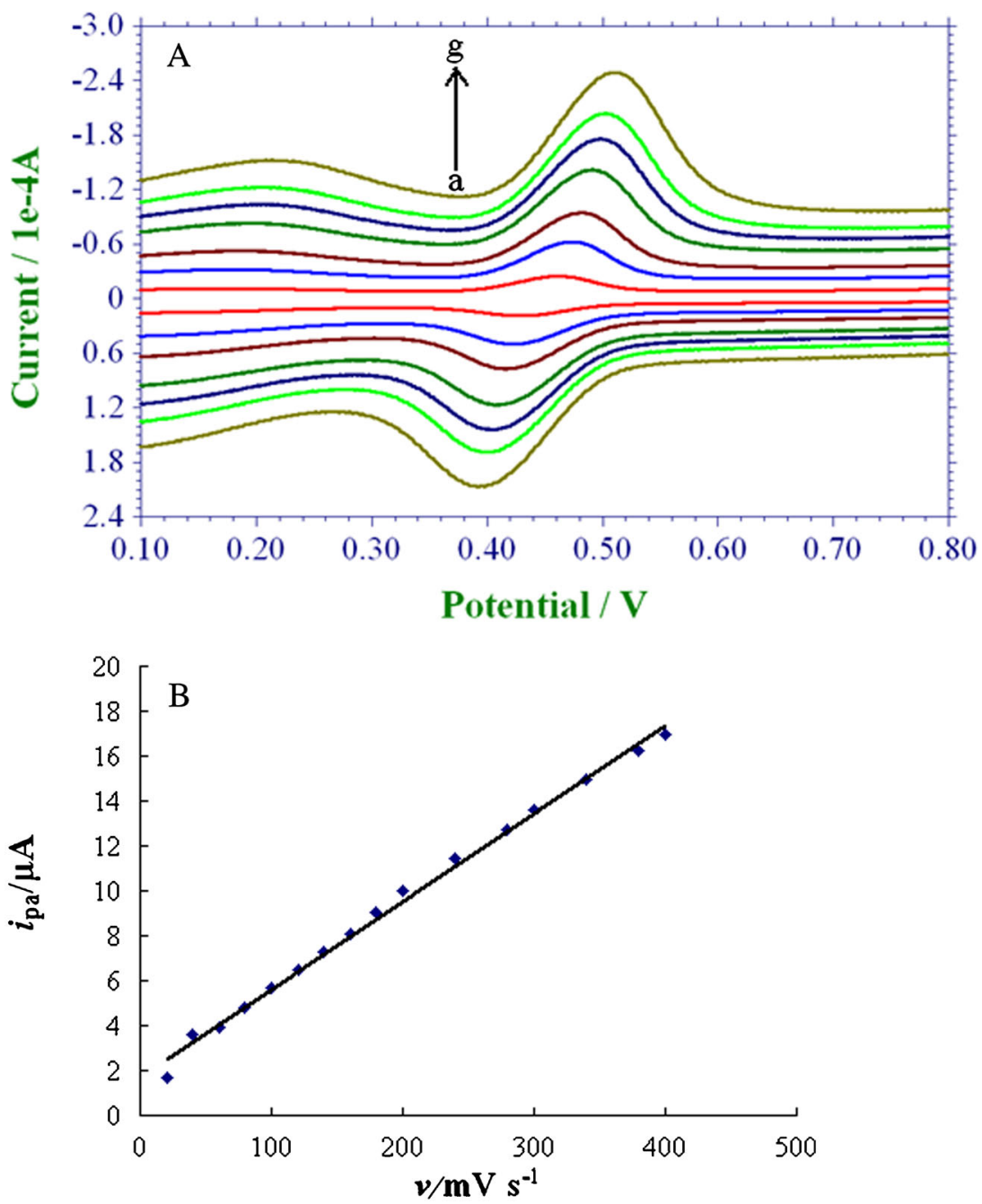

Figure 6. (a) $\mathrm{CVs}$ of $8.0 \times 10^{-5} \mathrm{M}$ rutin solution on the ERGOMWNTs/GCE at different scan rates (a-g: 20, 60, 100, 160, 200, 240, $\left.300 \mathrm{mV} \mathrm{s}^{-1}\right)$ in $0.1 \mathrm{M} \mathrm{PBS}(\mathrm{pH}=4.0)$; (b) plots of the oxidation peak current versus the scan rate. 
on the ERGO-MWNTs/GCE surface due to - $\mathrm{COOH}$, $-\mathrm{OH}$, and $-\mathrm{O}_{2} \mathrm{H}$ surface functional groups. As far as physisorption is concerned, it may be that rutin is physically adsorbed on the ERGO-MWNTs/GCE surface through some interactions, such as hydrophobic effects, van der Waals forces, $\pi \pi$ bonds, electrostatic action and hydrogen bonds. However, different mechanisms may act simultaneously; the prediction of rutin adsorption on ERGO-MWNTs/GCE is not straightforward. ${ }^{33}$ They need more detailed and thorough studies. According to the equation: $i_{\mathrm{pa}}=$ $n^{2} F^{2} v A \Gamma_{0}^{*} / 4 R T=n F Q v / 4 R T,{ }^{34} n$ was calculated to be $1.88 \approx 2$, which suggested that the oxidation process of rutin involved two electrons transfer. In addition, when the scan rate rose, the redox peak potentials shifted with the increasing peak-to-peak separation, which demonstrated that the electrode reaction gradually became more irreversible.
The ERGO-MWNTs/GCE can be used for the determination of rutin for 40 cycles (the current relative error $<10 \%$.). After each measurement, the three-electrode system was installed in a blank solution, and then magnetically stirred for $5 \mathrm{~min}$. Subsequently, the cyclic voltammetry scan was repeated successively until the redox peaks of rutin didn't appear for renewing the electrode.

\subsection{Effect of $p H$ on the response of rutin at the ERGO-MWNTS/GCE}

Figure 7 shows the effect of different $\mathrm{pH}$ on the response of $8.0 \times 10^{-5} \mathrm{M}$ rutin. When the $\mathrm{pH}$ changed from 3.0 to 9.0 , the anodic peak moved to the negative direction. There was a linear relationship between
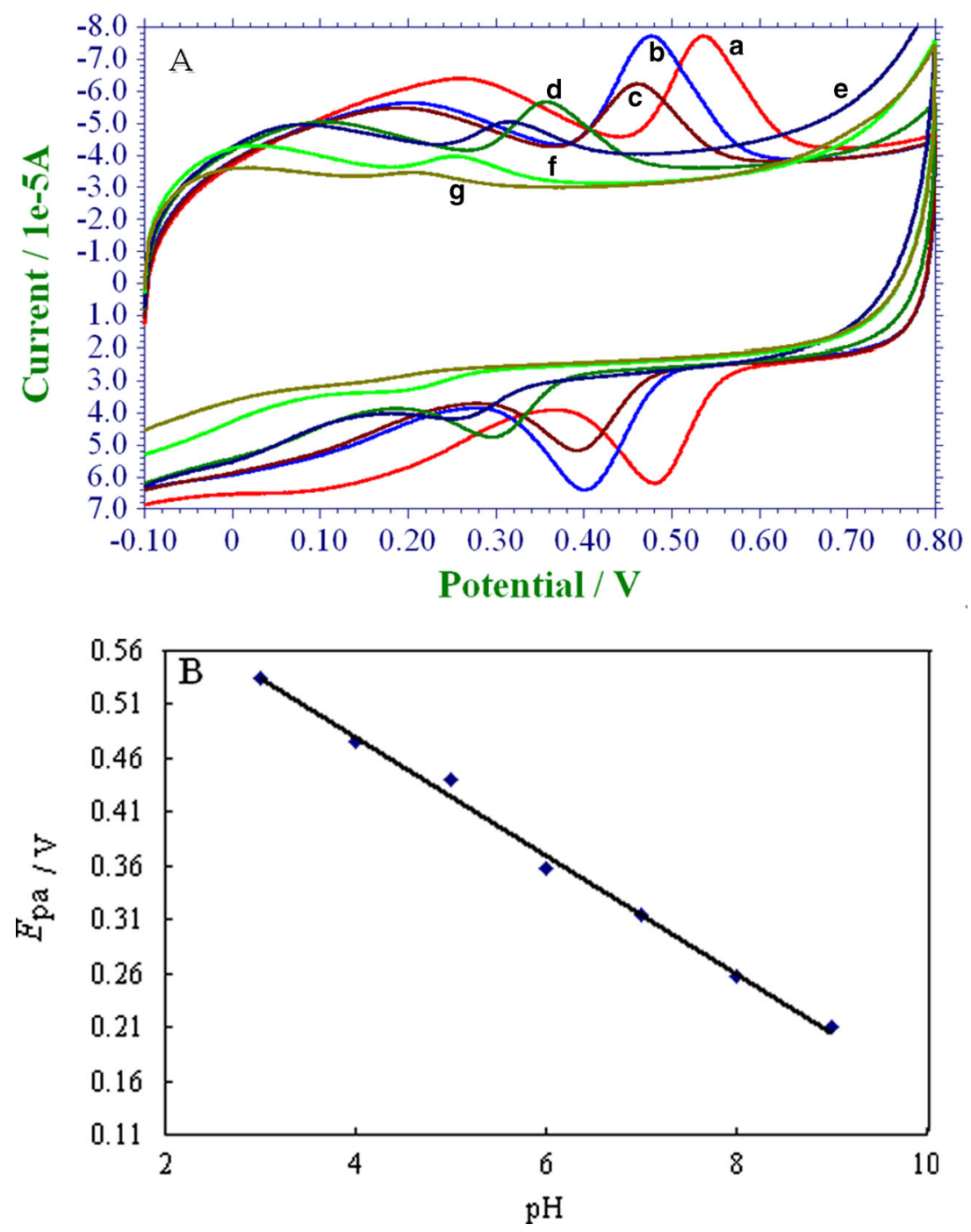

Figure 7. (a) Influence of solution $\mathrm{pH}$ on voltammograms of $8.0 \times 10^{-5} \mathrm{M}$ rutin with a scan rate of $80 \mathrm{mV} \mathrm{s}^{-1} .0 .1 \mathrm{M}$ phosphate buffer solution $\mathrm{pH}(\mathrm{a} \rightarrow \mathrm{g})$ : 3.0, 4.0, 5.0, 6.0, 7.0, 8.0, 9.0; (b) The linear relationship of $E_{\mathrm{pa}}$ versus $\mathrm{pH}$. 
the anodic peak potential and the $\mathrm{pH}$ value as follows: $E_{\mathrm{pa}} / \mathrm{V}=0.635-0.061 \mathrm{pH}(r=0.9946)$. According to the slope of $61 \mathrm{mV} \mathrm{pH}^{-1}$, it could be deduced that the number of electron and proton transferred were equal in the electrochemical reaction. With the increase in $\mathrm{pH}$, the peak current increased until at about $\mathrm{pH} 4.0$, and then decreased. But when the $\mathrm{pH}$ was over 8.0, the anodic peak became very small. These experimental phenomena were related to the proton involved in the electrochemical reaction. When $\mathrm{pH}$ exceeded 7.0, the electrostatic repulsion occurred between the ERGOMWNTs and rutin with negative charges. Therefore, acidic solution of $\mathrm{pH} 4.0$ was selected for the determination of rutin.

\subsection{Calibration curve}

As DPV has a higher current sensitivity and better resolution from background current than $\mathrm{CV}$, it was used for the determination of rutin. Figure 8 displayed the DPV of the different concentration of rutin at the ERGOMWNTs/GCE. The experimental results showed that $i_{\mathrm{pa}}$ was linear with the concentration of rutin in the ranges of $0.06-1.0 \mu \mathrm{M}$ and $1.0-20.0 \mu \mathrm{M}$ (figure 8). The regression equations were: $i_{\mathrm{pa}} / \mu \mathrm{A}=13.139 C_{\text {rutin }} / \mu \mathrm{M}$ $+0.4599(r=0.9973)$ for the range of $0.06-1.0 \mu \mathrm{M}$ and $i_{\mathrm{pa}} / \mu \mathrm{A}=1.985 C_{\text {rutin }} / \mu \mathrm{M}+12.624(r=0.9989)$ for the range of 1.0-20.0 $\mu \mathrm{M}$. The lowest detection concentration of rutin was $0.04 \mu \mathrm{M}$.

The repeatability and stability are the vital properties for the modified electrode, which should be studied for analytical measurement. The same ERGO-MWNTs/GCE was used for five successive measurements, and then the relative standard deviation (RSD) of the peak current was $6.3 \%$ for $1.0 \times$ $10^{-5} \mathrm{M}$ rutin. Moreover, five freshly prepared ERGOMWNTs/GCEs were used to measure $1.0 \times 10^{-5} \mathrm{M}$ rutin in the same condition, and then a RSD of $9.2 \%$ was obtained. These results revealed that the ERGOMWNTs/GCE was highly repeatable. The stability of the ERGO-MWNTs/GCE was investigated by determining the steady-state response current of $1.0 \times$ $10^{-5} \mathrm{M}$ rutin every day after preparation, the RSD of steady-state response current was $6.0 \%$. When it was not in use, the sensor was stored in $0.1 \mathrm{M}$ PBS $(\mathrm{pH}$ 7.0) at $4{ }^{\circ} \mathrm{C}$. The experimental results showed that the steady-state response current only decreased by $14 \%$ after 7 days, indicating that the ERGO-MWNTs/GCE electrode was considerably stable.

\subsection{Interference studies}

The influence of potential coexistent interference compounds were examined by this proposed method. A fixed amount of $2.0 \times 10^{-5} \mathrm{M}$ rutin was mixed with various foreign species. The results demonstrated that glucose, $\mathrm{K}^{+}, \mathrm{Cl}^{-}$and $\mathrm{SO}_{4}^{2-}$ were 500 times more than that of rutin, aminoacetic acid 200 times, $\mathrm{Zn}^{2+}$, $\mathrm{Ca}^{2+}$ and $\mathrm{Mg}^{2+} 50$ times, no observable interference was observed in the electrochemical response of rutin according to the relative error $< \pm 10 \%$.

Ascorbic acid (AA) is an electroactive substance, and always coexists in the Compound Rutin Tablets. At conventional electrodes, the oxidation peak potential of AA is very close to that of rutin, implying a poor selectivity for the measurement of AA and rutin in real samples.

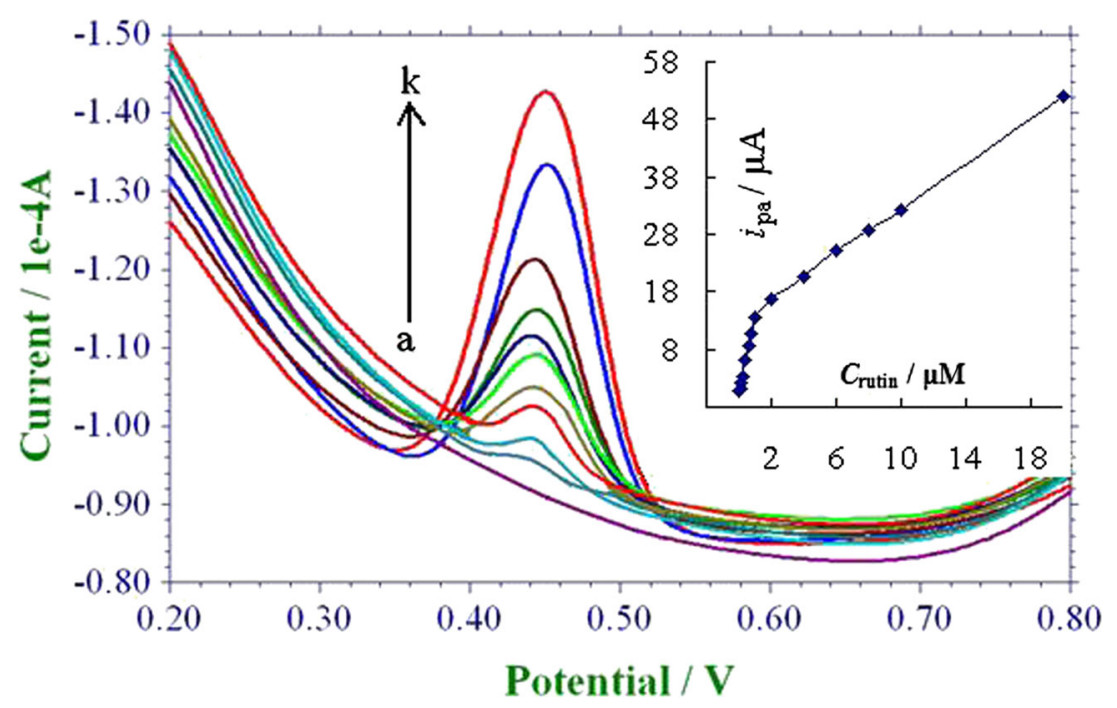

Figure 8. DPV of different concentrations of rutin $(\mathrm{a} \rightarrow \mathrm{k}): 0,0.06,0.08,0.1$, $0.2,0.4,0.6,0.8,1.0,2.0,4.0,6.0,8.0,10.0,20.0 \mu \mathrm{M}$; insert is the calibration curve of $i_{\text {pa }}$ vs. $C_{\text {rutin }}$. 


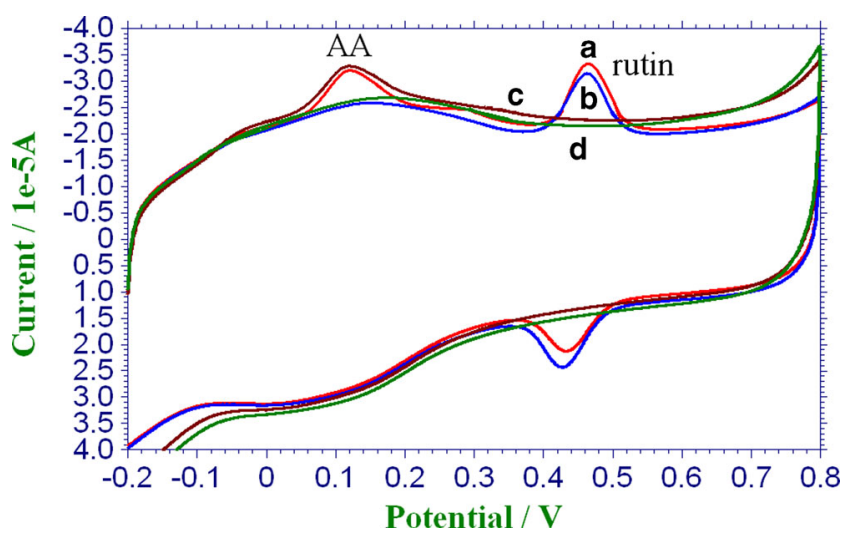

Figure 9. Cyclic voltammograms of the mixed solution of (a) $1.0 \times 10^{-4} \mathrm{M} \mathrm{AA}$ and $2.0 \times 10^{-5} \mathrm{M}$ rutin, (b) $2.0 \times$ $10^{-5} \mathrm{M}$ rutin, (c) $1.0 \times 10^{-4} \mathrm{M} \mathrm{AA},(\mathbf{d})$ and $0.1 \mathrm{M}$ phosphate buffer solution (pH 4.0) on the ERGO-MWNTs/GCE.

So, it is significant to develop more sensitive, selective and simple techniques for the segregative measurement of AA and rutin. Figure 9 displayed the CVs of the mixture of $2.0 \times 10^{-5} \mathrm{M}$ rutin and $1.0 \times 10^{-4} \mathrm{M}$ AA (a), $2.0 \times 10^{-5} \mathrm{M}$ rutin (b), $1.0 \times 10^{-4} \mathrm{M} \mathrm{AA}(\mathrm{c})$ and without AA and rutin (d) in $0.1 \mathrm{M} \mathrm{PBS}(\mathrm{pH} 4.0)$ at a ERGO-MWNTs/GCE. Figure 9c displayed an obvious anodic peak of AA at $0.117 \mathrm{~V}$. Figure 9a showed two anodic peaks at around $0.117 \mathrm{~V}$ and $0.467 \mathrm{~V}$, which were attributed to the oxidation of AA and rutin with a $0.350 \mathrm{~V}$ separation of both peaks, indicating broad enough separation for the simultaneous electrochemical determinations of rutin and AA in the mixed solution. In addition, in the presence of $1.0 \times 10^{-4} \mathrm{M}$ AA, the redox peak currents of rutin were almost not changed (figure 9a), which suggested that the ERGOMWNTs/GCE could eliminate the interference of AA for the determination of rutin.

\subsection{Analysis of real samples}

In order to examine the practicability of the proposed method, the content of rutin in Compound Rutin Tablets and Rutin Tablets were analyzed.The total value of rutin were obtained to be $19.1 \pm 0.23 \mathrm{mg}$ and $18.3 \pm 0.61 \mathrm{mg}$ per tablet for the Compound Rutin Tablets and Rutin Tablets, respectively (the labeled quantity of Rutin is was $20 \mathrm{mg}$ per tablet), which was calculated by multiplying the examined value and the diluted factor. In order to evaluate the correctness of the determination results, the standard addition method was employed to determine the ruin sample spiked with suitable ruin. The experimental results were listed in table 1 . In order to verify the accuracy of the proposed method, HPLC was used to determine the contents of rutin in real samples. By using HPLC, the contents of rutin were $19.3 \pm 0.49 \mathrm{mg}$ and $20.0 \pm 0.25 \mathrm{mg}$ per tablet for the Compound Rutin Tablets and Rutin Tablets, respectively, which were in agreement with the results obtained by the proposed electrochemical method. The experimental results indicated that the proposed electrochemical method could be efficiently used for Rutin determination in real samples.

\section{Conclusions}

In this paper, an ERGO-MWNTs/GCE was fabricated conveniently and firmly. Under the optimum condition, the ERGO-MWNTs/GCE provided excellent sensitivity and selectivity towards rutin. The anodic peak current was proportional to the rutin concentration in the ranges of $0.06-1.0 \mu \mathrm{M}$ and $1.0-20.0 \mu \mathrm{M}$ with the detection limit of $0.04 \mu \mathrm{M}$. The coexisting substance of AA declared no interference to the determination of rutin. The proposed method was further applied to the determination of rutin in the Compound Rutin Tablet and Rutin Tablet samples with satisfactory results.

\section{Acknowledgements}

The authors gratefully acknowledge the financial support from Nature Science Foundation of Anyang Normal University and National Natural Science Foundation of China (NSFC, No. 21102005).

Table 1. Determination results for rutin in Compound Rutin Tablets* and Rutin Tablets*.

\begin{tabular}{|c|c|c|c|c|c|}
\hline Sample & Detected (mg/tablet) & $\begin{array}{c}\text { Spiked } \\
\left(10^{-7} \mathrm{M}\right)\end{array}$ & Found $\left(10^{-7} \mathrm{M}\right)$ & $\begin{array}{c}\text { Average } \\
\text { recovery }(\%)\end{array}$ & $\begin{array}{c}\text { RSD of } \\
\text { recovery }(\%)\end{array}$ \\
\hline \multirow[t]{2}{*}{ Compound Rutin Tablets ${ }^{\mathrm{a}}$} & \multirow[t]{2}{*}{$19.3 \pm 0.49$} & 0.8 & $0.8 \pm 0.10$ & 100.0 & 6.0 \\
\hline & & 8.0 & $9.1 \pm 0.05$ & 113.7 & 4.5 \\
\hline \multirow[t]{2}{*}{ Rutin Tablets $^{\mathrm{a}}$} & \multirow[t]{2}{*}{$18.3 \pm 0.61$} & 0.8 & $0.76 \pm 0.22$ & 95.0 & 3.2 \\
\hline & & 8.0 & $8.2 \pm 0.62$ & 102.5 & 5.4 \\
\hline
\end{tabular}

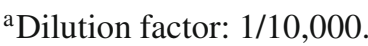

*Number of samples assayed: 5 . 


\section{References}

1. Chua L S 2013 J. Ethnopharmacol. 150805

2. Soni H, Malik J and Singhai A K 2013 Asin. J. Chem. 258371

3. Mendes Junior L G, Oliveira Monteiro M M and Carvalho A S 2013 Appl. Physiol. Nutr. Metab. 381099

4. Sharma S, Ali A, Ali J, Sahni J K and Baboota S 2013 Expert. Opin. Investig. Drugs. 221063

5. Zhang F, Qi X, Zou M and Li J 2013 J. Chem. 2013, http://dx.doi.org/10.1155/2013/324294.

6. Cheng C, Huang Y, Wang J, Zheng B, Yuang H and Xiao D 2013 Anal. Chem. 852601

7. Zeng H, Liang H, You J, Li S and Sui G 2013 Chin. J. Lumin. 34369

8. Satinsky D, Jaegerova K and Havlikova L 2013 Anal. Method. 61353

9. Leng H Q, Guo Y D and Liu W 2013 Guang. pu. 33 1801

10. Cai F, Xia J F and Wang Z H 2013 Adv. Mater. Res. 641 580

11. Liu M, Deng J and Chen Q 2013 Biosens. Bioelectron. 41275

12. Pumera M, Ambrosi A, Bonanni A, Chng E L K and Poh H L 2010 Trend. Anal. Chem. 29954

13. Li D, Muller M B, Gilje S, Kaner R B and Wallace G G 2008 Nat. Nanotechnol. 3101

14. Delgado J C, Herrera J M R, Jia X T and Cullen D A 2008 Nano. Lett. 82773

15. Stankovich S, Dikin D A, Piner R D and Kohlhaas K A 2007 Carbon 451558

16. Shao Y Y, Wang J, Engelhard M and Wang C M 2010 J. Mater. Chem. 20743
17. Guo H L, Wang X F, Qian Q Y and Wang F B 2009 ACS. Nano. 32653

18. Zhou M, Wang Y L, Zhai Y M and Zhai J F 2009 Chem. Eur. J. 156116

19. Ramesha G K and Sampath S 2009 J. Phys. Chem. C. 1137985

20. Iljima S 1991 Nature 35456

21. Lu P and Hsieh Y L 2010 ACS. Appl. Mater. Interfaces. 22413

22. Seo S D, Hwang I S, Lee S H and Shim H W 2012 Ceram. Int. 383017

23. Chen X, Zhu J, Xi Q and Yang W 2012 Sens. Actuators. B 161648

24. Hummers W S and Offeman R E 1958 J. Am. Chem. Soc. 801339

25. Qu L B, Yang S L and Li G 56 Electrochimi. Acta. 2934.

26. Stobinski L, Lesiak B, Kover L and Toth J 2010 J. Alloys. Compd. $\mathbf{5 0 1} 77$

27. Guo H L, Wang X F, Qian Q Y, Wang F B and Xia X H 2009 ACS. Nano. 32653

28. Mani V, Devadas B and Chen S M 2013 Biosens. Bioelectron. 41309

29. Prathisha K P, Barsana M M, Geng D, Sun X and Bretta C M A 2013 Electrochimi. Acta. 114533

30. Zhao C, Zheng W, Wang X, Zhang H, Cui X and Wang H 2013 Sci. Rep. 32986

31. Liu Y, Niu W F and Xu F 2011 Chin. J. Anal. Chem. 39 1676

32. Kumar A S and Swetha P 2010 Langmuir. 266874

33. Pan B and Xing B S 2008 Environ. Sci. Technol. 429005

34. Bard A J and Faulkner L R 1980 Electrochemical methods, fundamentals and applications (New York: Wiley \& Sons) 